\title{
A Biochemiluminescent Assay for Rapid Diagnosis of Influenza
}

\section{Xuexiang Lin}

Shenzhen Institutes of Advanced Technology Chinese Academy of Sciences

Jia Gu ( $\sim 21919013 @ q q . c o m$ )

Shenzhen Institutes of Advanced Technology Chinese Academy of Sciences https://orcid.org/00000001-7498-9892

\section{Research Article}

Keywords: Biochemiluminescence, Influenza, Luciferin, Luciferase, Neuraminidase, Instrument

Posted Date: October 14th, 2021

DOI: https://doi.org/10.21203/rs.3.rs-966400/v1

License: (1) This work is licensed under a Creative Commons Attribution 4.0 International License. Read Full License

Version of Record: A version of this preprint was published at Clinical and Experimental Medicine on January 10th, 2022. See the published version at https://doi.org/10.1007/s10238-021-00778-w. 


\section{Abstract}

Background: A biochemiluminescent assay of influenza diagnosis is presented. The assay diagnoses influenza based on detection of the influenza viral neuraminidase activity. An instrument designed for the assay is also reported. This assay solves the problem that current influenza virus diagnosis assays are susceptible to virus mutation.

Methods: A luciferase-based complex is synthesized as biochemiluminescent substrate. The substrate is cleaved to free luciferin with presence of influenza neuraminidase in specimen. Luciferase is oxidized to oxyluciferin with luciferin as catalyzer resulting in luminescence, which is proportional to the neuraminidase activity and measured by instrument. The instrument uses a photomultiplier tube (PMT) as sensor, with 24 test channels. Fine optical arrangements enable the instrument with high sensitive and accuracy.

Results: A total of 389 clinical specimens were collected to evaluate the performance of the assay in clinical settings. This assay had a sensitivity and specificity of $95.92 \%$ (95\% confidence interval: 91.38\%-98.12\%) and $97.93 \%$ (95\% confidence interval: $95.26 \%-99.11 \%)$, respectively, compared to the colloidal gold assay.

Conclusion: As a biochemiluminscence assay, this assay is advantageous in sensitivity and specificify. It does not require any washing and separation steps, which makes the instrument simple in design and easy to operate or maintenance. The assay is suitable for the rapid diagnosis of influenza virus in POC settings.

\section{Introduction}

Influenza is caused by influenza virus infection. Influenza A, B, and C are 3 types of viruses responsible for illness. Infection produced by the influenza viruses $A$ and $B$ ranges from mild respiratory illness like chills, headache, persistent cough, fatigue, to fatal pneumonia, myocarditis and heart failure [1].

The benefit of influenza treatment is greatest when antiviral therapy is started within 24 hours of symptom onset, so a reliable diagnostic method enabled rapid detection of influenza is essential [2,3]. At present, viral culture, antigen detection and polymerase chain reaction (PCR) are main conventional methods for influenza diagnosis. However, viral culture and PCR is tedious and time-consuming, and antigen detection is less sensitivity [4]. A comparison of several antigen detection assays to PCR revealed that sensitivities of immunoassays was only 60 to $80 \%$ for seasonal influenza virus stains [5-11].

Neuraminidase (NA) is one of the essential enzymes of influenza virus, which help progeny influenza virus cleave sialic acid and release from the host cell [2]. Therefore, NA plays an important role in virus replication and transmission $[12,13]$. All type $A$ and $B$ influenza viruses carry NA, so NA can be an ideal marker for diagnosis of influenza $[14,15]$. Literature studies have shown that, $\mathrm{N}$-acetylneuraminic acid (NeuAc) can be recognized by NA. In addition, 4,7-di-Omethyl-NeuAc has been revealed as specific 
receptor of NA for influenza A and B with excellent specificity. In this paper, we introduce an assay, the homogeneous biochemiluminescent assay (HBA), for rapid diagnosis of influenza by detecting viral NA activity $[16,17]$.

\section{Material And Methods}

\subsection{Substrate synthesis}

In this assay, we use a luciferase-based biochemiluminescent substrate, luciferin-4,7-di-0-methylneuraminic acid [18]. In the presence of influenza virus in a reaction, the substrate is cleaved to free luciferin, which becomes an active substrate of firefly luciferase. In the presence of firefly luciferase, the free luciferase is oxidized to oxyluciferin, resulting in a stable luminescent signal that can be detected by instruments $[19,20]$.

The process of substrate synthesis has been reported previously [16]. The synthesis of luciferin-4,7-di-0methyl-neuraminic acid is based on NeuAc and D-luciferin. The 4,7-di-0-methyl-2,8,9-tri-0-acetyl NeuAc methyl ester was achieved with optimized reaction conditions. Then the 2-chloro-4,7-di-0-methyl-8,9-diacetyl NeuAc methyl ester was prepared by chlornation reaction. Finally, the D-luciferin had been linked to 2-chloro-4,7-di-0-methyl-8,9-di-0-acetyl NeuAc methyl ester by Williamson ether synthesis, followed by removing the protection groups. The product purified by HPLC with its yield above $98 \%$.

The test kit of the HBA contains a sample buffer and a master mixture including the NA substrate, lucifery $1 \mathrm{~N}$-acteyl-neuraminic acid, and luciferase. Innovative reagent production technology makes the reagent as a freeze-dried bead, this allows for room temperature shipment and a longer shelf life.

\subsection{Clinical samples preparation and detection}

In the clinical study, a total of 389 clinical samples were collected between 26 January 2019 to 7 April 2019 at ZhuJiang Hospital of Southern Medical University, Guangzhou, China. Nasopharyngeal swabs were used to collect samples. The colloidal gold reagents were from Guangzhou Wondfo Biotech Co., Ltd., (registration number: 20153400613, production batch number: W05980202). PCR reagents and instrument were from Shanghai ZJ Bio-Tech Co.,Ltd.(registration number: 20173404656, production batch number: $P$ 20190601)

To perform a clinical sample testing, place the swab into sample buffer for elution. Add 250uL sample buffer to reagent tube to dissolve the reagent beads, and then incubate for 15 minutes in the instrument. The instrument will measure the luminescent signal and prints the results automatically. For samples that were not in sample buffer, dilute the samples 1:1 with sample buffer and then test as described above.

\subsection{Data Analyses}

The Sensitivity and specificity were used to determine the diagnostic yield among difference assays. 95\% confidence intervals were calculated according to the Wilson score method. Kappa was used to evaluate 
the consistency of the assays.

\subsection{Design of instrument}

Here we designed a compact and reliable instrument to measure luminescent signal emitted by the reaction mixture. The instrument consists of a reaction vessel, a heating apparatus, a measuring module, and a reaction vessel moving mechanism (Figure 1). The reaction vessel has 24 channels for reagents incubation, reaction and luminescence. The moving mechanism moves the vessel and bring particular channel to detection position. A photomultiplier tube (PMT) is used to capture the luminescent light. Optical fiber for transferring the luminescence light to PMT is located below the detection position and forms a confined space shielded from external light with reaction vessel. A metal cover for PMT protects it from vibration, moisture and external bright light, which may cause permanently damages.

The instrument is capable of carrying out measurement of luminescence quickly and effectively. The optical signals measured by the instrument indicated the quantification of influenza virus. The results of the instrument are read as Relative Light Unit (RLU).

\section{Results}

In order to calculate the appropriate cut-off value, 460 clinical specimens were tested, and the specimens were cultured for virus as well. The true positive rate (TPR) and false positive rate (FPR) were calculated under different cut-off values. The ROC curve is shown in Figure 2 . Since the instrument focuses on clinical screening, in this study, 220K RLU was used as the cut-off value. The TPR, FPR were $66.04 \%$, $7.86 \%$, respectively.

To evaluate linearity and linear range, we tested samples of influenza virus strains (A/CA/07/2009; wild type) with concentrations of 2.83 to $5.06 \log T_{C I D}{ }_{50} / \mathrm{ml}$. The correlation coefficient $\left(\mathrm{R}^{2}\right)$ was $0.9967(95 \%$ confidence interval: 0.9690-1.0)

To evaluate the variability, two positive samples and one negative sample were tested 4 times a day for 12 consecutive days. The results (Table 1 ) indicated that HBA could make correct diagnoses of these samples.

To evaluate the limit of detection (LOD), we tested 5 samples of influenza virus strains A/CA/07/2009 and $A / N C / 37 / 2009$ for 20 times. The concentrations of the samples were close to the detection limit. The results are shown in Table 2. The diagnostic accuracy of positive influenza samples should be at least $95 \%$, so the detection limits of $A / C A / 07 / 2009$ and $A / N C / 37 / 2009$ were $995 \mathrm{TCID}_{50} / \mathrm{mL}$ and 953 $\mathrm{TCID}_{50} / \mathrm{mL}$, respectively.

To evaluate the performance of the assay in clinical settings, a total of 389 clinical samples were collected. These studies were approved by the medical ethics committee at ZhuJiang Hospital of Southern Medical University and conducted according to the requirements of the China Food and Drug 
Administration (CFDA). We used nasopharyngeal swabs to collect samples, and the test results were recorded and saved according to relevant guidelines and regulations.

Of 389 samples, 147 were diagnosed positive and 242 negative by colloidal gold assay. 146 were diagnosed positive and 243 negative by HBA. The results of 11 samples were inconsistent under these two assays. After retesting with PCR, 6 samples were consistent with the results of HBA, and 5 samples were consistent with the colloidal gold's. The results are presented in Table 3. Overall, the HBA had a sensitivity and specificity of $95.92 \%$ (95\% confidence interval: $91.38 \%-98.12 \%)$ and $97.93 \%$ (95\% confidence interval: $95.26 \%-99.11 \%)$, respectively. The Kappa value was $0.94(95 \%$ confidence interval: 0.905-0.975)

\section{Discussion And Conclusion}

Influenza viruses are the most prevalent pathogens that cause acute respiratory tract infections, a rapid and sensitive diagnosis assay is essential to elucidate accurately any outbreak due to influenza virus. High mutation rate and large number of variants make the diagnosis of influenza facing many challenges [21]. Based on the existing research NA is a conserved enzyme of influenza virus, which is not susceptible to virus mutation, so it is an ideal influenza diagnostic marker [22].

As a biochemiluminscence assay, HBA is advantageous in sensitivity and specificity. In addition, the catalyzer and substrate of HBA does not exist in the human specimens, and the analyte is captured in a liquid phase without using micro-particles, so HBA does not requires any washing and separation steps that have obvious influence on measurement [23]. The HBA performs on simple and small instruments with a detection time less than 15 minutes, and clinical samples can be tested in batches because the instrument is designed with 24 channels. These advantages contribute to more timely results and higher laboratory workflow efficiency.

The HBA has the advantages, but also it has some limitations, such as unable to identify subtypes of influenza viruses. For example, surveillance laboratories are more demanding and require comprehensive analysis of the virus includes knowledge of the virus's genetic makeup and antigenic type/subtype information, which are unachievable by HBA. Especially, influenza $C$ does not carry NA, so the HBA cannot be used for influenza $\mathrm{C}$ diagnosis.

At present, the instrument and reagent is applying for registration certificate in China Food and Drug Administration (CFDA). Future work is needed to expand the detection of viral pathogens by such rapid methods.

\section{Declarations}

\section{Funding}

This research has been supported by the China National Key R\&D Program (Grant No. 2020YFC2003800) 


\section{Conflicts of interest}

The authors did not receive support from any organization for the submitted work.

\section{Availableility of data and material}

All the data released to this work are available at the corresponding author.

\section{Code availability}

Not applicable.

\section{Ethics approval}

The experimental protocol was established, according to the ethical guidelines of the Helsinki Declaration and was approved by the medical ethics committee at ZhuJiang Hospital of Southern Medical University.

\section{Consent for publication}

Patients signed informed consent regarding publishing their data.

\section{Consent to participate}

Informed consent was obtained from all individual participants included in the study. Informed consent was obtained from legal guardians.

\section{Acknowledgments}

We are very grateful to Zhujiang Hospital of Southern Medical University for providing detection samples.

\section{Author Contributions}

Xuexiang Lin: methodology, investigation, analysis, writing. Jia Gu: supervision, resources, review and editing, project administration.

\section{References}

1. Coiras MT, Pérez-Brea P, García M, Casas I. Simultaneous detection of influenza A, B, and C viruses, respiratory syncytial virus, and adenoviruses in clinical samples by multiplex reverse transcription nested-PCR assay. J Med Virol. 2003;69(1):132-44.

2. Wen F, Wan XF. Influenza Neuraminidase: Underrated Role in Receptor Binding. Trends Microbiol. 2019;27:477-9.

3. Gaitonde DY, Moore FC, Morgan MK. Influenza: Diagnosis and Treatment. Am Fam Physician. 2019;100(12):751-8. 
4. Mahony JB. Detection of Respiratory Viruses by Molecular Methods. Clin Microbiol Rev. 2008;21(4):716-47.

5. Jannetto PJ, Buchan BW, Vaughan KA, et al. Real-time detection of influenza a, influenza B, and respiratory syncytial virus a and $B$ in respiratory specimens by use of nanoparticle probes. $J$ Clin Microbiol. 2010;48(11):3997-4002.

6. Chang X, Liu D, Wei H, et al. A Study on the Rapid Detection Technology of Influenza A Virus Based on Fluorescence Immunochromatography. Journal of Chengdu Medical College. 2017.

7. Controlprevention C. Evaluation of rapid influenza diagnostic tests for detection of novel influenza $A$ (H1N1) virus-United States, 2009. MMWR Morb Mortal Wkly Rep. 2009;58:826-9.

8. Crawford JM, Stallone R, Zhang F, et al. Laboratory Surge Response to Pandemic (H1N1) 2009 Outbreak, New York City Metropolitan Area, USA. Emerg Infect Dis. 2010;16(1):8-13.

9. Ginocchio CC, Zhang F, Manji R, et al. Evaluation of multiple test methods for the detection of the novel 2009 influenza A (H1N1) during the New York City outbreak. J Clin Virol. 2009;45(3):191-5.

10. Shawn Vasoo JS, Kamaljit Singh. Rapid antigen tests for diagnosis of pandemic (Swine) influenza A/H1N1. Clin Infect Dis. 2009;49(7):1090-3.

11. Webster RG, Govorkova EA. Continuing challenges in influenza. Ann N Y Acad Sci. 2014;1323(1):115-39.

12. Air GM. Influenza neuraminidase. Influenza Other Respiratory Viruses. 2012;6(4):245-56.

13. Sylte MJ, Suarez DL. Influenza Neuraminidase as a Vaccine Antigen. Current Topics in Microbiology Immunology. 2009;333:227-41.

14. Gao Z, Niikura M, Withers SG. Ultrasensitive Fluorogenic Reagents for Neuraminidase Titration. Angew Chem Int Ed. 2017;56:6112-6.

15. Hl'asová Z, Košík I, Ondrejovič M, Miertuš S, Katrlík J. Methods and Current Trends in Determination of Neuraminidase Activity and Evaluation of Neuraminidase Inhibitors. Crit Rev Anal Chem. 2018;49:350-67.

16. Hui KM, Li XJ, Pan L, Li XJ, editors. A homogeneous biochemiluminescent assay for detection of influenza2015 May 01, 2015.

17. Wu S, Lin X, Hui KM, Yang S, Li XJ. A Biochemiluminescent Sialidase Assay for Diagnosis of Bacterial Vaginosis. Sci Rep. 2019;9(1):1-9.

18. Isojima Y, Isoshima T, Nagai K, Kikuchi K, Nakagawa H. Ultraweak biochemiluminescence detected from rat hippocampal slices. Neuroreport. 1995;6(4):658-60.

19. Wettey FR, Jackson AP. Luciferase reporter assay. Subcell Biochem. 2006;40(40):423-5.

20. Hemmati R, Hosseinkhani S, Sajedi RH, et al. Luciferin-Regenerating Enzyme Mediates Firefly Luciferase Activation Through Direct Effects of D-Cysteine on Luciferase Structure and Activity. Photochemistry Photobiology. 2015;91(4):828-36.

21. Banerjee D, Kanwar N, Hassan F, Essmyer C, Selvarangan R. Comparison of Six Sample-to-Answer Influenza A/B and RSV Nucleic Acid Amplification Assays Using Respiratory Specimens from 
Children. J Clin Microbiol. 2018;56(11):e00930-18.

22. Marjuki H, Mishin VP, Sleeman K, et al. Bioluminescence-based neuraminidase inhibition assay for monitoring influenza virus drug susceptibility in clinical specimens. Antimicrobial Agents Chemotherapy. 2013;57(11):5209-15.

23. Khalil OS, Zurek TF, Genger KR, Pepe CJ, Yi-Her J, Cotter SM, inventorsMethod. and apparatus for heterogeneous chemiluminescence assay 1995.

\section{Tables}

Table 1 Repeatability of different samples

\begin{tabular}{|c|c|c|c|c|c|c|}
\hline & \multirow{2}{*}{\multicolumn{3}{|c|}{$\begin{array}{l}\text { Positive } \\
\text { Sample } 1\end{array}$}} & \multirow{2}{*}{\multicolumn{2}{|c|}{$\begin{array}{l}\text { Positive } \\
\text { Sample } 2\end{array}$}} & \multirow{3}{*}{$\begin{array}{l}\text { Negative } \\
\text { Sample } \\
1\end{array}$} \\
\hline & & & & & & \\
\hline & 1 & 2 & 3 & 1 & 2 & \\
\hline Mean RLU(n = 48) & 373 & 1009 & 3933 & 724 & 3357 & 101 \\
\hline SD & 82 & 198 & 1003 & 228 & 352 & 34 \\
\hline$\% \mathrm{CV}$ & 21.91 & 19.68 & 25.50 & 31.49 & 10.48 & 33.59 \\
\hline \% Positive & 100 & 100 & 100 & 100 & 100 & 0 \\
\hline
\end{tabular}

Table 2 LOD at various concentrations of influenza virus strains

\begin{tabular}{|llllll|}
\hline & \multicolumn{2}{l}{$\mathrm{A} / \mathrm{CA} / 07 / 2009$} & \multicolumn{3}{l|}{$\mathrm{A} / \mathrm{NC} / 39 / 2009$} \\
\cline { 2 - 6 } & 1 & 2 & 3 & 1 & 2 \\
\hline Concentrations (TCID50/mL) & 663 & 995 & 1,326 & 953 & 1,271 \\
\hline Mean(S/CO) & 0.98 & 1.58 & 1.96 & 1.15 & 1.87 \\
\hline$\%$ CV(S/CO) & 13.81 & 7.98 & 6.59 & 8.36 & 3.45 \\
\hline$\%$ Positive & $45 \%$ & $100 \%$ & $100 \%$ & $100 \%$ & $100 \%$ \\
\hline
\end{tabular}

Table 3 The test result of clinical study

\begin{tabular}{|lllll|}
\hline & & \multicolumn{2}{l}{ Results of colloidal gold } & total \\
\cline { 3 - 5 } & & Positive & Negative & \\
\hline \multirow{2}{*}{ Results of the HBA } & Positive & 141 & 5 & 146 \\
\cline { 2 - 5 } & Negative & 6 & 237 & 243 \\
\hline \multirow{2}{*}{ Total } & & 147 & 242 & 389 \\
\hline
\end{tabular}


Figures

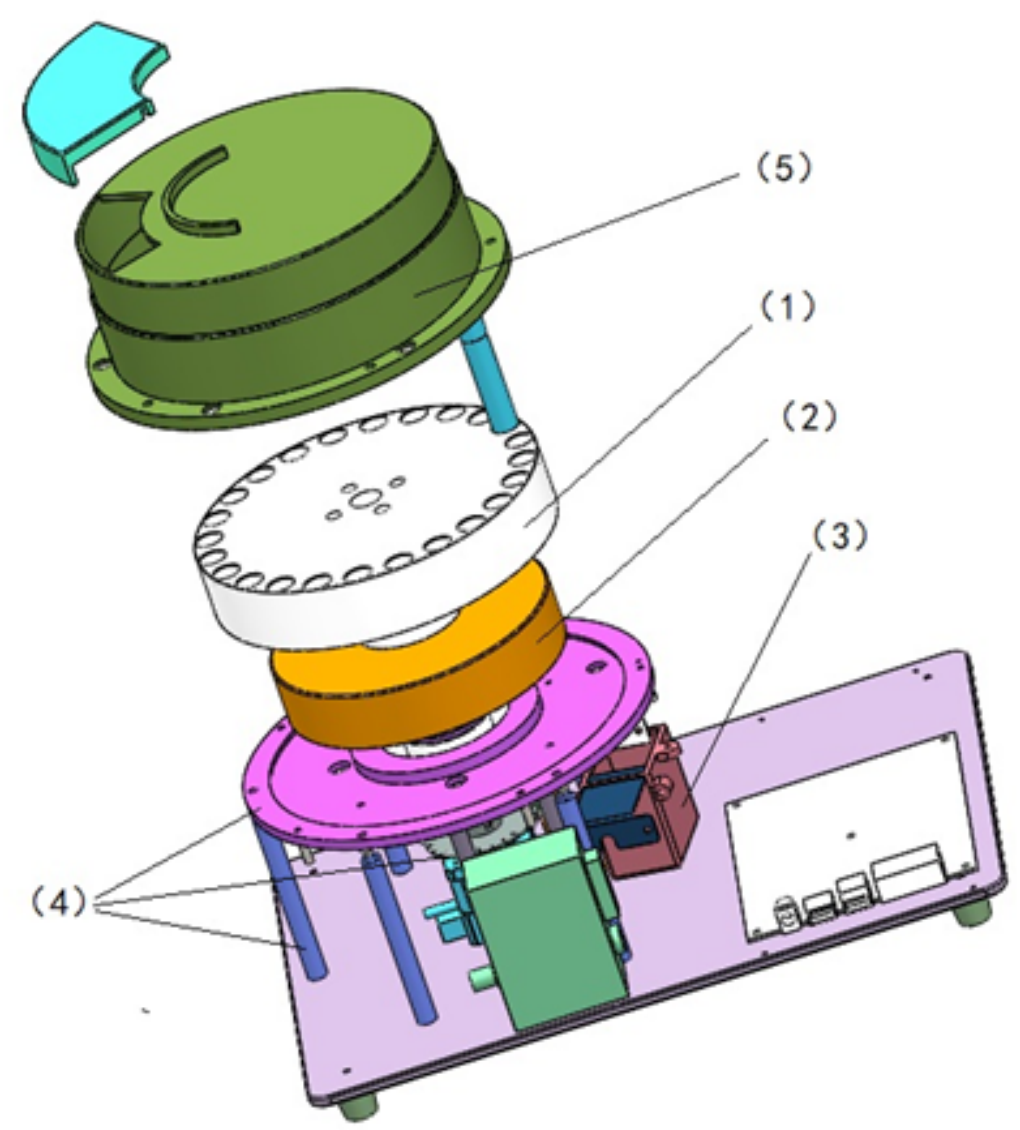

\section{Figure 1}

Instrument structure diagram (1هReaction vessel; (2) heating apparatus; (3) measuring module; (4) moving apparatus; (5) light-shielding cover 


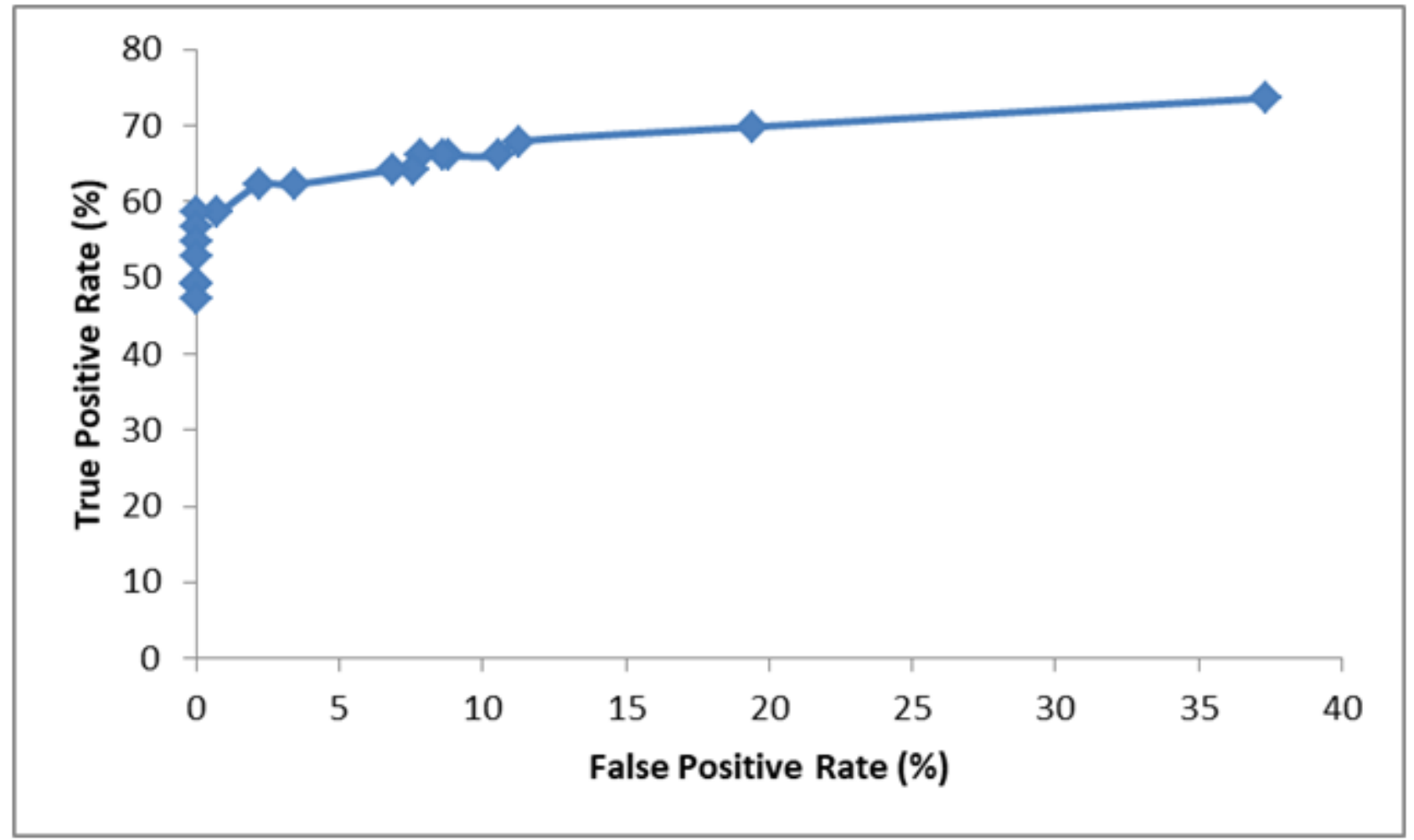

Figure 2

The ROC curve of 460 clinical specimens 